This item was submitted to Loughborough's Research Repository by the author.

Items in Figshare are protected by copyright, with all rights reserved, unless otherwise indicated.

\title{
Application of response surface methodology to maximize the productivity of scalable automated human embryonic stem cell manufacture
}

PLEASE CITE THE PUBLISHED VERSION

http://dx.doi.org/10.2217/RME.12.109

PUBLISHER

(c) Future Medicine Ltd

VERSION

VoR (Version of Record)

\section{LICENCE}

CC BY-NC-ND 4.0

\section{REPOSITORY RECORD}

Ratcliffe, Elizabeth, Paul C. Hourd, Juan-Jose Guijarro-Leach, Erin Rayment, David J. Williams, and Robert James Thomas. 2019. "Application of Response Surface Methodology to Maximize the Productivity of Scalable Automated Human Embryonic Stem Cell Manufacture”. figshare. https://hdl.handle.net/2134/13171. 
This item was submitted to Loughborough's Institutional Repository (https://dspace.lboro.ac.uk/) by the author and is made available under the following Creative Commons Licence conditions.

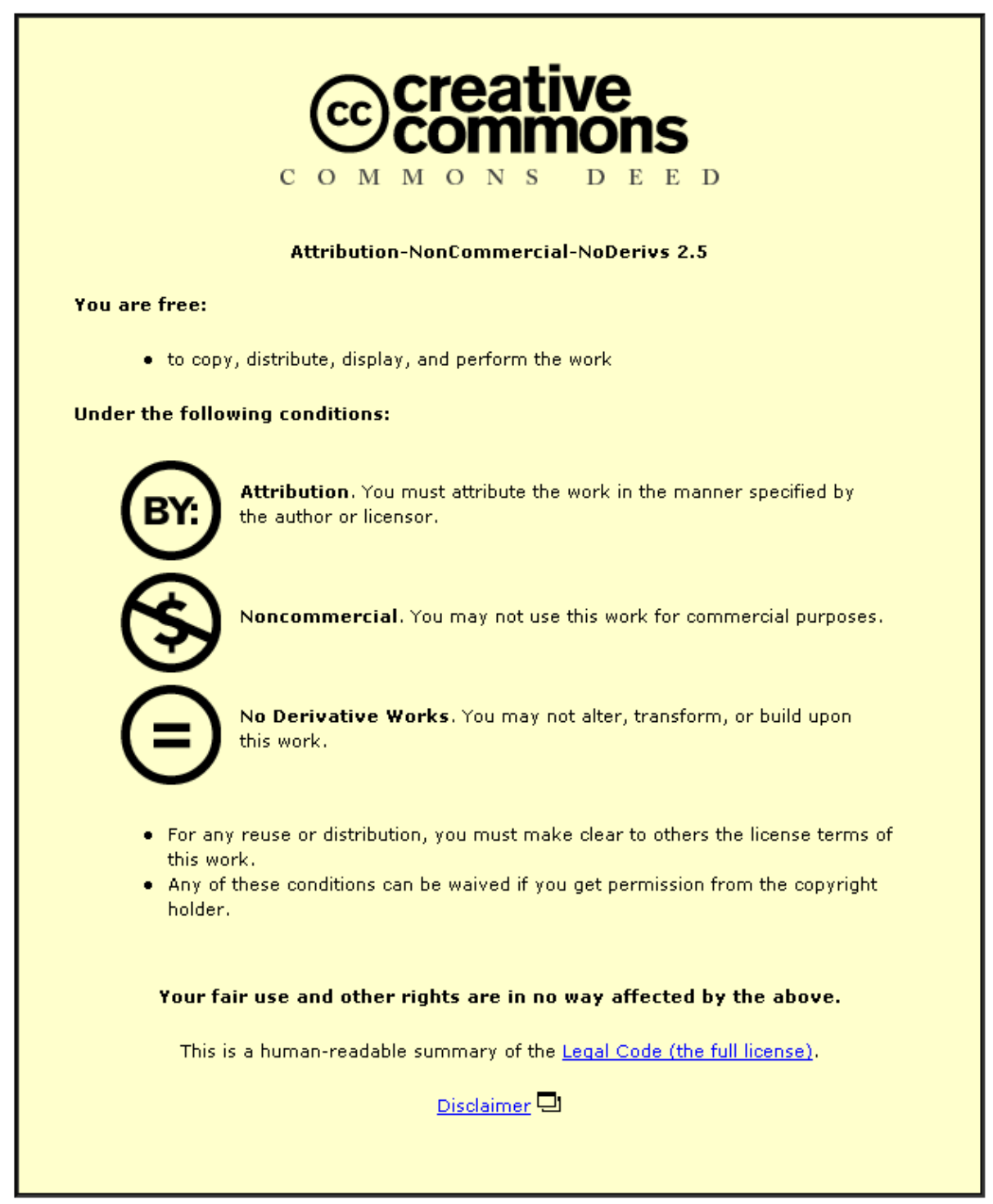

For the full text of this licence, please go to: http://creativecommons.org/licenses/by-nc-nd/2.5/ 


\title{
Application of response surface methodology to maximize the productivity of scalable automated human embryonic stem cell manufacture
}

\begin{abstract}
Aim: Commercial regenerative medicine will require large quantities of clinical-specification human cells. The cost and quality of manufacture is notoriously difficult to control due to highly complex processes with poorly defined tolerances. As a step to overcome this, we aimed to demonstrate the use of 'qualityby-design' tools to define the operating space for economic passage of a scalable human embryonic stem cell production method with minimal cell loss. Materials \& methods: Design of experiments response surface methodology was applied to generate empirical models to predict optimal operating conditions for a unit of manufacture of a previously developed automatable and scalable human embryonic stem cell production method. Results \& conclusion: Two models were defined to predict cell yield and cell recovery rate postpassage, in terms of the predictor variables of media volume, cell seeding density, media exchange and length of passage. Predicted operating conditions for maximized productivity were successfully validated. Such 'quality-by-design' type approaches to process design and optimization will be essential to reduce the risk of product failure and patient harm, and to build regulatory confidence in cell therapy manufacturing processes.
\end{abstract}

KEYWORDS: automation cost of goods human embryonic stem cell manufacture process control response surface methodology scalability

During recent years, there has been significant progress in the development of human cell-based therapies to treat a wide range of disease states [1]. Regenerative medicine (RM) approaches use such cells to repair or replace damaged or diseased human cells, tissues or organs in order to restore normal function [2]. The large number of RM cell products in preclinical or clinical development support projections for the development of a multibillion dollar industry over the coming decade, with long-term public health benefits [3]. However, manufacturing of cell therapies is complex and many face problems achieving the transition from the laboratory bench-scale to robust manufacturing-scale processes with consistent quality and commercially viable costs [4]. Much of this is due to a lack of economic and controllable manufacturing platforms for adherent cell production, poor definition of the critical process parameters that impact cell product quality and the difficulty of defining the quality of biological input materials; this is compounded by a product that is highly sensitive to changes in the production environment. Purification or enrichment of the end product to deal with process variability increases costs and is restricted relative to biomolecular therapies where the final product is distinct from the bioprocessed cells [5]. Many candidate cell-based therapy bioprocesses are therefore considered to be high risk with significant end-point testing and failure rates.

Other complex manufacturing industries have applied multivariate designs of experiments within a systematic framework to address similar process optimization and control issues [6,7]. These experimental designs provide empirical models that predict the response of critical process outputs to the levels of multiple input variables. Depending on the process response targeted, such process characterization can reduce risk by defining critical parameter operating windows and indicate opportunities to increase process efficiency or product quality. Compared with conventional 'one factor at a time' experimental approaches, they define interdependent effects of process inputs on process responses (prevalent in biomanufacture systems) and are highly efficient in terms of data output per experimental condition $[7,8]$. However, the complexity of cell culture bioprocesses and the confounding of input variable effects over time make them hard to model as a single unit. Design of experiments approaches can address this through focusing on subprocess units; a sequence of controlled subprocesses, or units of manufacture, will generate a controlled, continuous process. Systematic application of these methods needs to become routine in order to enable data-driven decision-making to improve RM process outputs

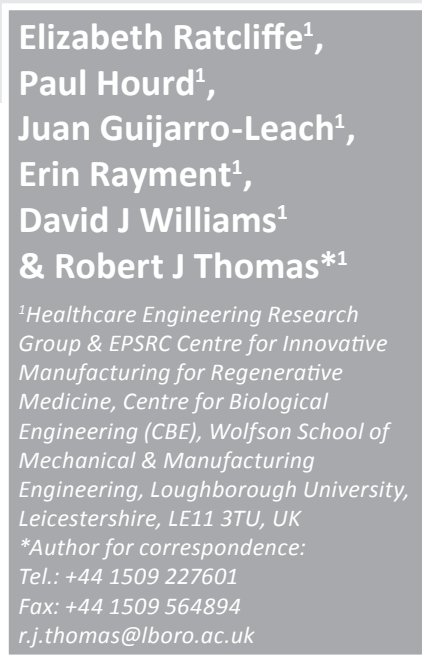

Regenerative Medicine 
that are critical to product quality and support risk-based approaches to manufacture. However, only early examples of practical applications, and the issues with these examples, are currently emerging in RM [9].

Human embryonic stem cells (hESCs) are of significant importance as a renewable source of cells for RM applications due to their ability to self-renew and differentiate into any cell type [10]. The generation of large numbers of cells for prospective therapies and hESC maintenance is labor intensive; for example, it is estimated that in excess of $10^{9} \mathrm{hESC}$ would be required to generate enough cardiomyocytes to repair an infarcted heart [11]. To address this issue, we previously developed a fully automated robotic process, using the CompacT SelecT ${ }^{\mathrm{TM}}$ platform (TAP Biosystems, UK; with a previously reported single-cell suspension passage method [12]), to provide proof of principle that highly controlled automated production of $10^{9} \mathrm{hESCs}$ is feasible [13].

We have used a modification of this scalable and automatable method of cell culture with defined mTeSR1 media to optimize and validate a single manufacturing subprocess (between two passages) for cost and quality. Cost is an important target because, unlike conventional small-molecule pharmaceuticals, cell-based product manufacture defines a significant proportion of cost of goods supplied and therefore commercial viability [3]. We selected media volume, media change frequency, cell seeding density and time between passage as significant determinants of process cost. We employed a response surface method (RSM) experimental design, which is a high-resolution method capable of modeling nonlinear relationships, to model the effects of these variables between two passages (the subprocess) on cell yield. We also modeled the effects of the input variables on the recovery of the cells after experimental conditions were applied (assessed by population doublings in $24 \mathrm{~h}$ postpassage) because of reports of cell death and poor recovery after single-cell passage methods [14]. This enabled validation of an RSM predicted economic production strategy for an industrially relevant and scalable hESC culture process.

\section{Materials \& methods}

\section{Materials \& general culture}

All tissue culture reagents were purchased from Invitrogen (UK), plastic ware from Fisher Scientific (UK) and chemicals from Sigma-Aldrich (UK), unless otherwise stated.
All work processes were controlled by a set of detailed standard operating procedures to minimize background variability, and laboratory environmental conditions were monitored (i.e., temperature) to ensure no deviations occurred that could impact the data.

\section{hESC culture}

To ensure experimentation was economic, but that results would be scalable in line with previously described automated protocols, we designed a low-scale T25-based manual process with direct step-by-step equivalence to the automated and scalable T175-based singlecell suspension passage method previously reported [13]. Specifically, the hESC line, H9 (WiCell Research Institute, WI, USA), was cultured in feeder-free conditions on growth factor-reduced hESC-qualified MatrigelTM (BD Biosciences, UK) in mTeSR1 medium (StemCell Technologies, France). Cells were thawed at passage 35 and passaged three times (under central point conditions; refer to TABLE 1) prior to experimental intervention. Cultures were maintained at $37^{\circ} \mathrm{C}, 5 \% \mathrm{CO}_{2}$ in a humidified atmosphere with medium change every $24 \mathrm{~h}$ and passaged every $48 \mathrm{~h}$ unless otherwise stated. Matrigel was used to coat $25-\mathrm{cm}^{2}$ Corning tissue culture flasks (T25s) according to manufacturer's instructions by dilution in cold DMEM-F12, allowing polymerization to occur at $22^{\circ} \mathrm{C}$ for $45 \mathrm{~min}$. Prior to use, medium was aspirated from flasks and the culture surface washed with phosphate-buffered saline. Culture passage was performed by incubation with $5 \mathrm{ml}$ TrypLETM SelecT (Life Technologies, NY, USA) for $3 \mathrm{~min}$ at $37^{\circ} \mathrm{C}$, coupled with shaking the flasks to liberate single cells and small cell aggregates, and trituration of warm $\left(37^{\circ} \mathrm{C}\right)$ mTeSR 1 across the flask surface was carried out to generate cell pools for analysis and further culture seeding. Cultures were initiated at several cell seeding densities and medium volumes (as detailed in Table 1; viability: $98.80 \pm 0.18 \%$; $\mu \pm \sigma, n=3)$.

\section{hESC analysis}

Cell pool density and viability assessment was performed using the automated Innovatis Cedex ${ }^{\text {TM }}$ Analyzer (Roche Diagnostics Ltd, UK) and the trypan blue dye exclusion method (Roche Diagnostics Ltd). The equation $\log _{10}$ (cell output/cell input) $/ \log _{10} 2$ was used to calculate population doublings of cultures. Flow cytometry for hESC pluripotency and early differentiation markers was performed using a Cell Lab 
Quanta $^{\mathrm{TM}}$ SC Flow Cytometer (Beckman Coulter, UK) and the following conjugated antibodies and appropriate isotype controls according to manufacturer's instructions; Oct4aphycoerythrin (PE), SSEA1-PE, SSEA4-PE, alkaline phosphatase-PE (R\&D Systems, UK), SSEA3-PE, Tra-1-81-PE and Nanog-PE (BD Biosciences, UK). Data presented are the means and standard deviations of percentage marker expression for triplicate samples $(\mu \pm \sigma, n=3)$.

\section{RSM design \& analysis}

A three-level, three-factor (i.e., $3^{3}$ ) Box-Behnken experimental design was applied to evaluate the effects of three factors - seeding density $\left(\mathrm{X}_{\mathrm{SD}}\right)$, media volume $\left(\mathrm{X}_{\mathrm{MV}}\right)$ and media exchange time $\left(\mathrm{X}_{\mathrm{FT}}\right)$ - in the period between two consecutive passages. hESC expansion performance at the second passage, and $24 \mathrm{~h}$ thereafter (harvest), were analyzed as response variables $\left(\mathrm{Y}_{1}\right.$ and $\mathrm{Y}_{2}$, respectively). This design studied the combined effects of the three factors in a single block of 12 sets of test conditions, augmented with five central points to estimate pure error. Three levels were attributed to each factor, coded as low (-1), medium (0) and high (+1) (Table 1). The order of the experiments was fully randomized. Additionally, the RSM study considered process time as a fourth factor, by applying the $3^{3}$ Box-Behnken design across three levels of duration between the first and second passage (standard every $48 \mathrm{~h}$ ).

Statistical analysis was performed with the software package Design Expert ${ }^{\circledR}$, version 8.0.5 (Stat-Ease, Inc., MN, USA). Analysis of variance
Table 1. Standard and response surface method experimental culture conditions.

\begin{tabular}{|c|c|c|c|}
\hline \multirow[t]{2}{*}{ Factors } & \multicolumn{3}{|c|}{ Levels } \\
\hline & $\operatorname{Low}(-1)$ & Medium (0) & $\operatorname{High}(+1)$ \\
\hline$X_{S D}\left(10^{6}\right.$ cells $)$ & 1.50 & $2.50^{+}$ & 3.50 \\
\hline $\mathrm{X}_{\mathrm{MV}}(\mathrm{ml})$ & 5.00 & $8.75^{\dagger}$ & 12.50 \\
\hline$X_{F T}(h)$ & 24 & $24+50 \%^{\dagger}$ & $24+100 \%$ \\
\hline Process time (h) & 36 & $48^{+}$ & 60 \\
\hline \multicolumn{4}{|c|}{$\begin{array}{l}\text { Thirteen factorial combinations of these culture parameter levels, specified by the response surface } \\
\text { method experimental design software, were conducted on experimental laboratory cultures to } \\
\text { generate the data for the process models. Because culture duration was different for each of the three } \\
\text { response surface method experiments, the time of media exchange is not specified as an absolute } \\
\text { value in the table. Media exchange occurred at } 24 \mathrm{~h} \text {, halfway between } 24 \mathrm{~h} \text { and the end of the culture } \\
\text { period (specified as } 24+50 \% \text { ) or there was no media exchange (specified as } 24+100 \%) \text {. The } \\
\text { absolute time value of the exchange therefore varied depending on the length of the culture period. } \\
{ }^{+} \text {Central point culture conditions. } \\
X_{F T} \text {. Media exchange time; } X_{M V} \text {. Media volume; } X_{S D} \text { : Seeding density (T25 flask). }\end{array}$} \\
\hline
\end{tabular}

was applied to establish a prediction model for each response. The selected models were evaluated according to a battery of adequacy tests and graphical analysis was carried out as previously described [15], and in brief below. Each fitted model was examined to ensure that it provides an adequate approximation to the true system in order to empirically assess the usefulness of the predictive capabilities of the model and to verify that the model satisfies the assumptions of the analysis of variance.

In brief, the regression model diagnostics for reported models were within acceptable limits (summary statistics are shown in Tables $2 \& 3$ ). Lack-of-fit tests were not significant, indicating that additional variation in the residuals could not be removed with a better model. The linear correlation plots between the actual and

Table 2. A reduced two-factor interaction model that describes the response surface for the process response passage yield.

\begin{tabular}{|c|c|c|c|c|c|}
\hline Source & Sum of squares & df & Mean square & F value & $p$-value \\
\hline Model & 65.63 & 3 & 21.88 & 37.38 & $<0.0001$ \\
\hline$X_{S D}$ & 61.94 & 1 & 61.94 & 105.84 & $<0.0001$ \\
\hline $\mathrm{X}_{\mathrm{MV}}$ & 0.66 & 1 & 0.66 & 1.13 & 0.3071 \\
\hline $\mathrm{X}_{\mathrm{SD}} \mathrm{X}_{\mathrm{MV}}$ & 3.03 & 1 & 3.03 & 5.17 & 0.0405 \\
\hline Residual & 7.61 & 13 & 0.59 & - & - \\
\hline Lack of fit & 6.74 & 9 & 0.75 & 3.44 & 0.1229 \\
\hline Pure error & 0.87 & 4 & 0.22 & - & - \\
\hline Cor total & 73.23 & 16 & - & - & - \\
\hline \multicolumn{6}{|c|}{$\begin{array}{l}\text { The experimental data were used to generate theoretical models of process performance around the experimental levels } \\
\text { run in the laboratory. The table shows a summary of the analysis of variance data and model statistics. } \\
\text { Standard deviation: } 0.76 ; \text { mean: } 6.56 ; C V: 11.67 ; \text { PRESS: } 16.05 ; R^{2}: 0.8961 ; \text { Adj } R^{2}: 0.8721 ; \text { Pred } R^{2}: 0.7809 ; \\
\text { Adeq precision: } 19.686 \text {. } \\
\text { Adeq precision: Signal-to-noise ratio; Adj } R^{2} \text { : Amount of variation around the mean explained by the model, adjusted for } \\
\text { the number of terms in the model; Cor total: Corrected total of all information corrected for the mean; CV: Coefficient of } \\
\text { variation; df: Degrees of freedom; F: Fischer's variance ratio; Pred } R^{2}: \text { Amount of variation in new data explained by the } \\
\text { model; PRESS: Predicted residual error sum of squares; } R^{2}: \text { Amount of variation around the mean explained by the model; } \\
X_{M V}: \text { Media volume; } X_{S D} \text { : Seeding density. }\end{array}$} \\
\hline
\end{tabular}




\begin{tabular}{|c|c|c|c|c|c|}
\hline Source & Sum of squares & df & Mean square & F value & p-value \\
\hline Model & 127.95 & 6 & 21.33 & 39.35 & $<0.0001$ \\
\hline$X_{S D}$ & 33.73 & 1 & 33.73 & 62.25 & $<0.0001$ \\
\hline $\mathrm{X}_{\mathrm{MV}}$ & 35.91 & 1 & 35.91 & 66.26 & $<0.0001$ \\
\hline$X_{F T}$ & 13.14 & 1 & 13.14 & 24.25 & 0.0006 \\
\hline $\mathrm{X}_{\mathrm{SD}} \mathrm{X}_{\mathrm{MV}}$ & 28.85 & 1 & 28.85 & 53.23 & $<0.0001$ \\
\hline$X_{S D} X_{F T}$ & 3.16 & 1 & 3.16 & 5.84 & 0.0363 \\
\hline$X_{M V} X_{F T}$ & 13.16 & 1 & 13.16 & 24.29 & 0.0006 \\
\hline Residual & 5.42 & 10 & 0.54 & - & - \\
\hline Lack of fit & 3.90 & 6 & 0.65 & 1.71 & 0.3146 \\
\hline Pure error & 1.52 & 4 & 0.38 & - & - \\
\hline Cor total & 133.37 & 16 & - & - & - \\
\hline \multicolumn{6}{|c|}{$\begin{array}{l}\text { The experimental data were used to generate theoretical models of process performance around the experimental levels } \\
\text { run in the laboratory. The table shows a summary of the analysis of variance data and model statistics. } \\
\text { Standard deviation: } 0.74 ; \text { mean: } 6.69 ; C V: 11.01 ; \text { PRESS: } 18.22 ; R^{2}: 0.9594 ; \text { Adj } R^{2}: 0.9350 ; \text { Pred } R^{2}: 0.8634 ; \\
\text { Adeq precision: } 20.340 \text {. } \\
\text { 'The data were transformed to achieve an appropriate distribution characteristic for modeling (transformation: } \\
\text { power } \lambda: 2.11 ; \text { constant: } 2.4497) \text {. } \\
\text { Adeq precision: Signal-to-noise ratio; Adj } R^{2}: \text { Amount of variation around the mean explained by the model, adjusted for } \\
\text { the number of terms in the model; Cor total: Corrected total of all information corrected for the mean; CV: Coefficient of } \\
\text { variation; df: Degrees of freedom; F: Fischer's variance ratio; Pred } R^{2}: \text { Amount of variation in new data explained by the } \\
\text { model; PRESS: Predicted residual error sum of squares; } R^{2}: \text { Amount of variation around the mean explained by the model; } \\
X_{F T} \cdot \text { Media exchange time; } X_{M V} \text { Media volume; } X_{S D}: \text { Seeding density. }\end{array}$} \\
\hline
\end{tabular}

predicted response variables indicate coefficient of determination values close to 1 , confirming high predictive power for both models and a good fit between the model and the experimental data. The close agreement between the three coefficient of determination statistics indicates that there is good agreement between the experimental and predicted values for both responses. The precision of both models indicates that the signal-to-noise ratio of the data is adequate (>4) and confirms that the models can be used to navigate the design space. The coefficient of variation for both responses is low, indicating close agreement between repeat measurements.

Graphical residual and influence diagnostics were also conducted for the reported models and were within acceptable limits. Graphical plots were of internally studentized residuals versus: the predicted responses, showing random scatter and absence of trends, confirming constant variance; the experimental run order, showing random scatter, indicating the absence of sources of nonrandom error or lurking variables; or the individual factors, showing random scatter, indicating absence of curvature or significant differences in the magnitude of residuals between levels. The predicted versus measured response plots showed close correlation between the modeled and measured data points.
Graphical plots of the externally studentized residuals and Cook's distance versus the experimental run order did not detect any data points that could be considered to be outliers or otherwise unusual/suspect. The DFFITS and DFBETAS plots did not indicate any treatments with overly large influences on predictions or regression coefficients.

\section{Results}

\section{Unit of manufacture}

A standard 8-day cell expansion process from an H9 hESC cryobank was developed as an experimental unit of manufacture for application of the RSM tool. Prior to applying the RSM experimental conditions, the unit of manufacture was repeatedly performed under standard operating conditions to provide evidence of statistical control of the process, confirm hESC pluripotency marker expression (Figure 1 ) and to verify the performance of the measurement systems. Upon defrost, mean viable cell recovery was $5.5 \times 10^{6} \pm 0.3(\mu \pm \sigma$, $\mathrm{n}=3)$ and average viability was $98 \pm 0.3 \%(\mu \pm \sigma$, $\mathrm{n}=3$ ). At day 6 of the process, which was the point of the subsequent RSM experiment, average viable cell yield was $67.2 \times 10^{6} \pm 0.3(\mu \pm \sigma$, $\mathrm{n}=3$; 3.6 population doublings) and average viability was $99.5 \pm 0.3 \%(\mu \pm \sigma, \mathrm{n}=3)$, which is equivalent to early expansion levels that have 
been previously seen with the automated singlecell suspension passage method [13]. All cells showed typical monolayer hESC morphology, high expression levels of pluripotency indicators and low expression levels of differentiation indicators, as shown in Figure 1B \& 1C.

The objective of the RSM experiment was to reduce the cost of the unit of manufacture while maintaining or increasing cell product quality through targeting a reduction in process time and the use of expensive raw materials. A series of RSM cell culture experiments was therefore designed to minimize process time, cell culture liquid volumes and input cell numbers through optimization of the process parameters (factors) of $\mathrm{X}_{\mathrm{MV}}$, media exchange schedule $\left(\mathrm{X}_{\mathrm{FT}}\right)$ and $\mathrm{X}_{\mathrm{SD}}$.
Experimental data were used to model twoprocess responses: the yield of the cells from the experimental runs (cell yield $\left[\mathrm{Y}_{1}\right]$ ) and the ability of the cells to proliferate in the passage period after exposure to the conditions of the experimental run (population doublings at 24-h harvest postexperiment $\left.\left[\mathrm{Y}_{2}\right]\right)$.

\section{Model selection}

Initially, three Box-Behnken RSM cell culture experiments, incorporating the chosen factors at software-specified design levels, were conducted over 36-, 48- and 60-h passage periods (Table 1 ). The experimental data were initially used to model the cell recovery after the experimental passage (response variable $\mathrm{Y}_{2}$, assessed by cell

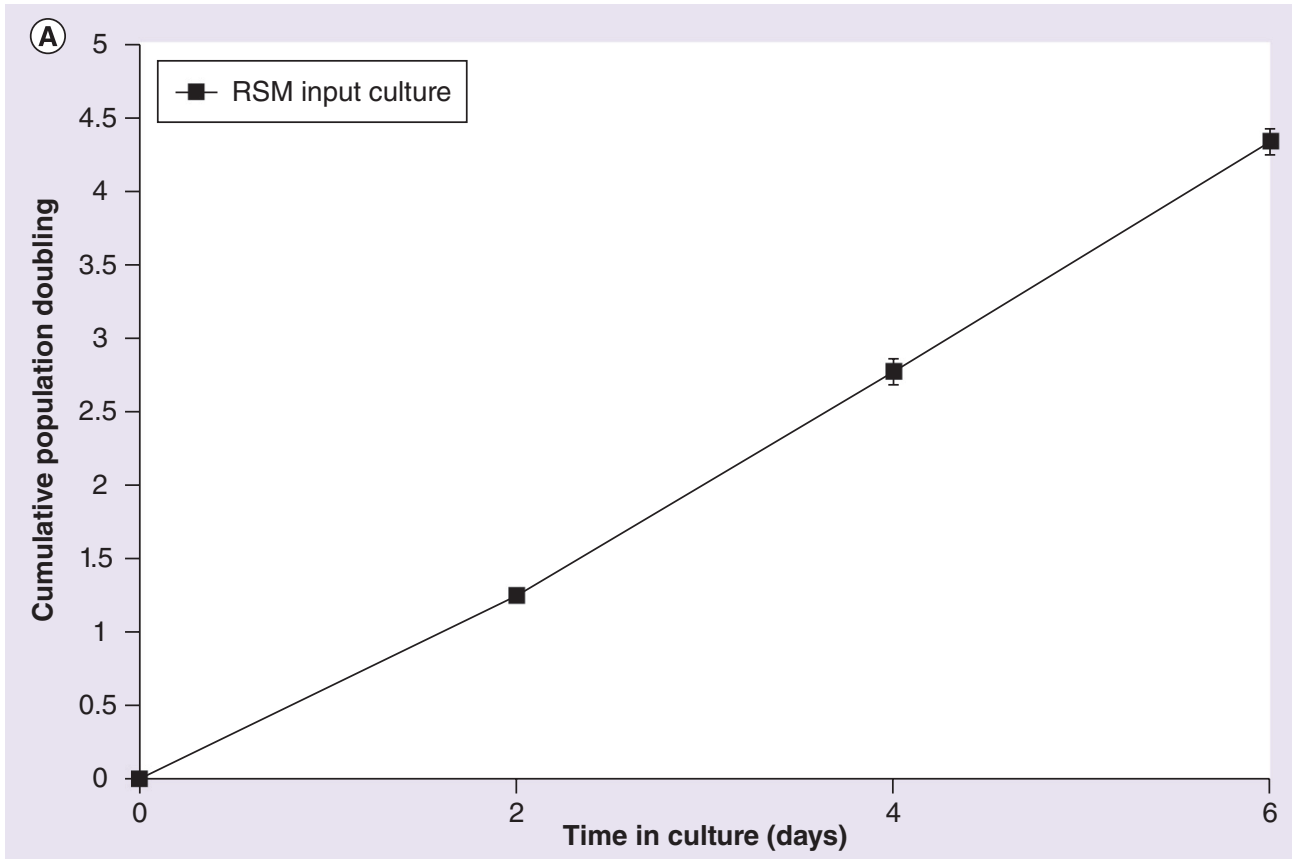

(B)

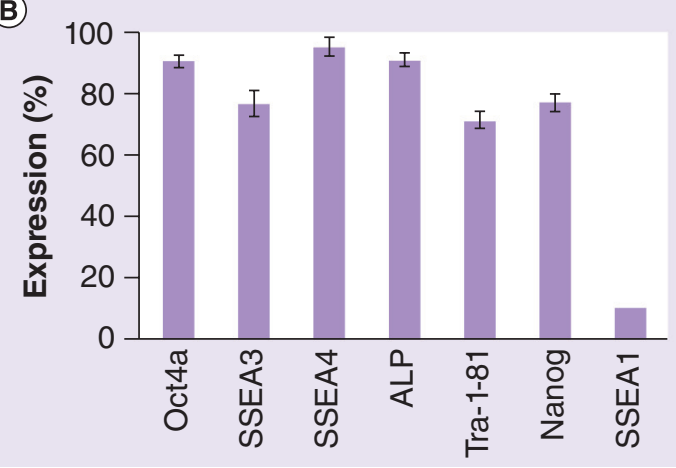

(C)

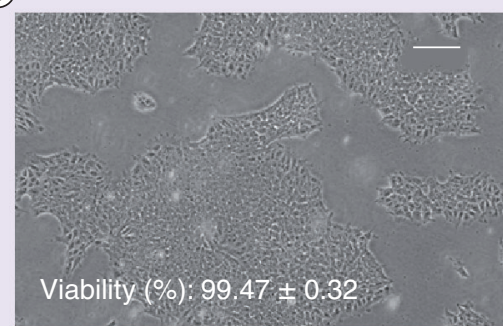

Figure 1. Baseline performance of the bioprocess used for the response surface method experimental designs. (A) Repeated processing of the unit of manufacture of human embryonic stem cells under central conditions (TABLE 1) over the experimental period and under strict operating procedures showed stable average cumulative population doublings over time $(\mu \pm \sigma, n=3)$.

(B \& C) After 6 days of expansion, at the point of experimental intervention, cells showed typical morphology, high viability and normal pluripotency marker expression levels.

Scale bar: $60 \mu \mathrm{m}$.

RSM: Response surface method. 
proliferation over the $24-\mathrm{h}$ postexperimental run). This was to exclude conditions that prohibited cell recovery postpassage due to the common recognition of this issue with single-cell passage methods [14]. Analysis of the models with a 48- and 60-h passage period did not provide outcomes that met critical optimization criteria. The 48-h model could not meet the threshold criteria of sufficient recovery in the 24 -h postexperimental condition period (minimum specified as $\leq 0.2$ population doublings). The $60-\mathrm{h}$ model did not give a large enough advantage in cell yield to offset the projected cost of near doubling of the culture time. Therefore, the 36-h model was selected for further analysis.

\section{Modeling the effects}

The design software was used to establish the best model to fit the cell culture experimental data. The 36-h model $Y_{1}$ and $Y_{2}$ were both best predicted by a reduced first-order model with interaction. The $\mathrm{Y}_{2}$ response required a power transformation. Attempts at model reduction by backward elimination of nonsignificant terms resulted in extremely minor improvements in the model for $\mathrm{Y}_{2}$ and, therefore, all model terms were included in the analysis of variance.

Both of the overall models reported were highly significant $(\mathrm{p}<0.0001)$, indicating significant factor effects and that the models can

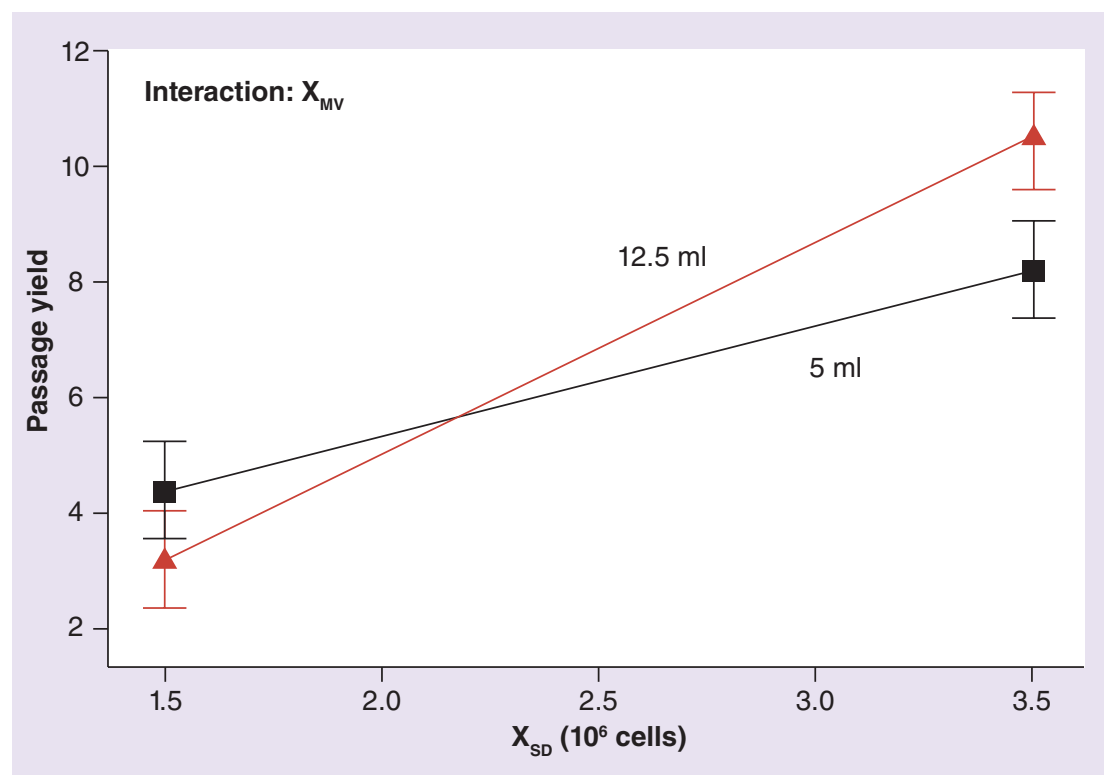

Figure 2. Two-factor interaction graph for the effect on cell yield of seeding density and media volume (media exchange does not affect the interaction). Bars at the end points of each line represent \pm half least squares difference at $\alpha=0.05$; nonoverlapping bars indicate that the means are significantly different. The graph shows that the yield relative to input cells is maximized at either a low $\mathrm{X}_{\mathrm{SD}^{\prime}}$ low $\mathrm{X}_{\mathrm{MV}}$ or at a high $\mathrm{X}_{\mathrm{SD}}$ and high $\mathrm{X}_{\mathrm{MV}}$. $\mathrm{X}_{\mathrm{MV}}$ : Media volume; $\mathrm{X}_{\mathrm{SD}}$ : Seeding density. be considered to be of sufficient quality to navigate the experimental design space and to predict new observations. The analysis of variance and model statistics confirmed the adequacy of the models and revealed significant model terms and coefficient estimates that describe the response surface for both $Y_{1}$ and $Y_{2}$ as a function of the factor settings (Taвles $2 \& 3$ ). The final predictive model equations in terms of the actual factors (rounded) were:

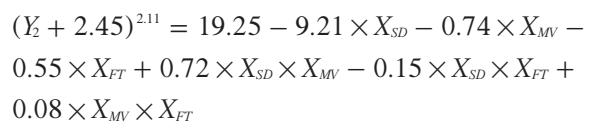

and

$Y_{1}=4.05+0.75 \times X_{S D}-0.50 \times X_{M V}+0.23 \times X_{S D} \times X_{M V}$

\section{Biomanufacture significance}

The model that describes the $Y_{1}$ response surface was simpler than that describing the $\mathrm{Y}_{2}$ response surface. It showed a significant linear effect of $\mathrm{X}_{\mathrm{SD}}$ and $\mathrm{X}_{\mathrm{MV}}$, with no significant effect of $\mathrm{X}_{\mathrm{FT}}$. There was also a significant interaction between these two factors: the increase in $\mathrm{Y}_{1}$ generated by an increased $X_{S D}$ is greater given a higher $X_{M V}$. This effect can be visualized in Figure 2, where it is shown only for one level of media exchange (as media exchange does not affect the process yield in this model). The practical significance of this is that, within the range of the model, to maximize the passage yield, the $\mathrm{X}_{\mathrm{SD}}$ needs to be balanced with the $\mathrm{X}_{\mathrm{MV}}$. Both high-density, low-volume and low-density, high-volume conditions only result in a twofold $\mathrm{Y}_{1}$ over input, whereas high-density, high-volume and low-density, low-volume conditions both give an approximatly threefold $Y_{1}$ over the same period. Mechanistically, this suggests a requirement to balance nutrient supply with concentrations of cell-secreted factors. It suggests that biomanufacture equipment that enables continual feed strategies may offer increased cell productivity with respect to time.

The model for $\mathrm{Y}_{2}$ showed significant linear effects of all three factors and also significant interactions between all factors. The behavior of the response as a function of the factor settings can be visualized graphically with the aid of response surface plots and a cube plot (Figure 3). Similarly to the $\mathrm{Y}_{1}$ model, these interactions have practical significance. Figure 3A shows that the cells will only recover quickly from low-mediavolume conditions in the experimental passage period if they have also had a low $\mathrm{X}_{\mathrm{SD}}$ and $\mathrm{X}_{\mathrm{FT}}$ (the former having more impact than the latter). Figure 3B shows that when the $\mathrm{X}_{\mathrm{SD}}$ is at a low value, 


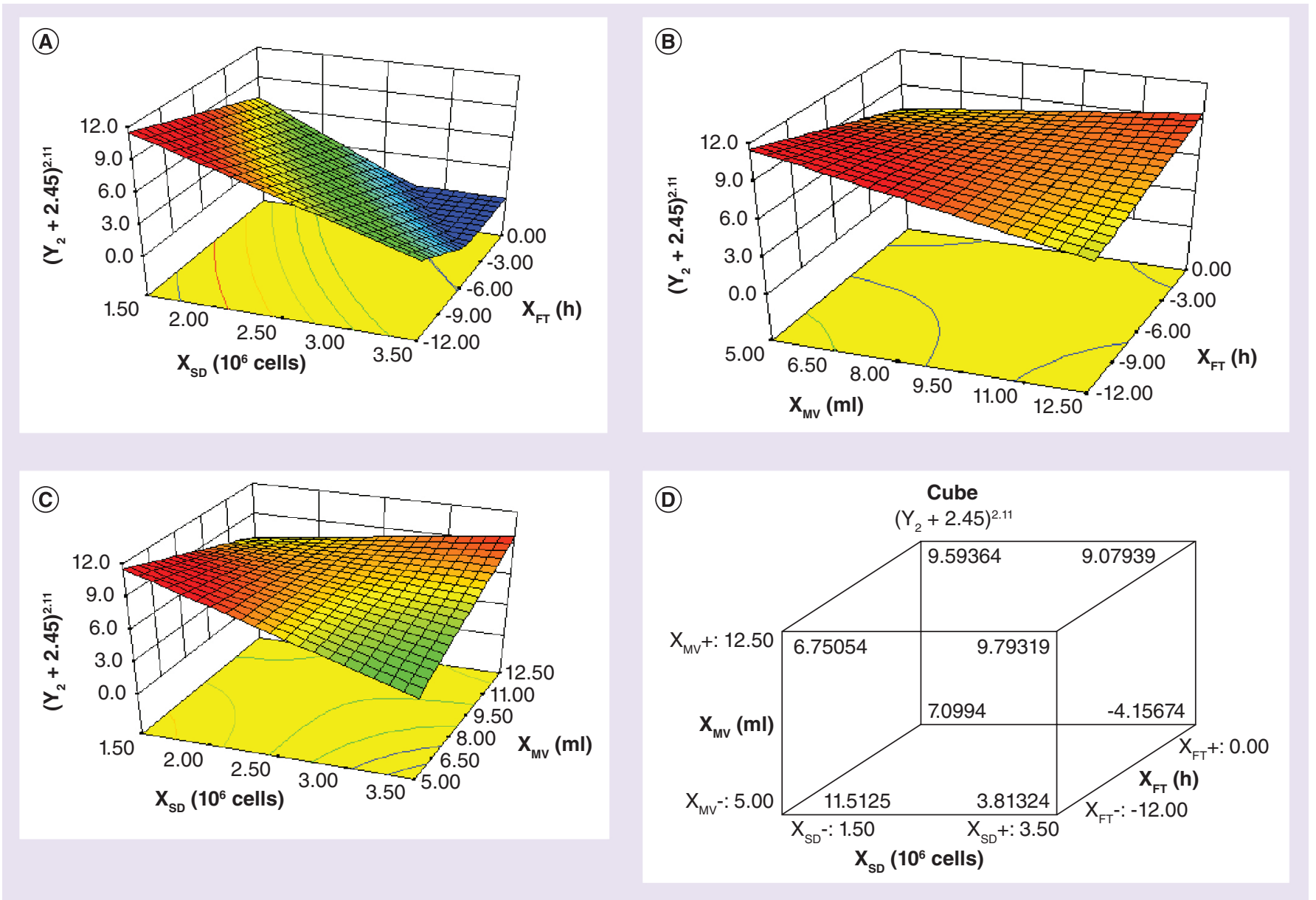

Figure 3. Response surface charts of process models. (A) 3D response surface plots for cell recovery ( $\mathrm{Y}_{2}$; note axis transformed) after experimental conditions as a function of $X_{S D}$ and $X_{F T}$ with $X_{M V}$ set at the low-level value (5 ml). Optimal cell recovery is clearly achieved after experimental conditions with low $X_{S D}$ and early media exchange. (B) $X_{M V}$ and $X_{F T}$ with $X_{S D}$ set at the low-level value. $Y_{2}$ is relatively insensitive to other factors (performing well) when $X_{S D}$ was low in the experimental period. (C) $X_{S D}$ and $X_{M V}$ with $X_{F T}$ set at the high-level value (-12; i.e., $24 \mathrm{~h})$. Cell recovery $\left(\mathrm{Y}_{2}\right)$ in the postexperimental period is reduced with either low $X_{M V}$ and high $X_{S D^{\prime}}$ or high $X_{M V}$ and low $X_{S D^{\prime}}$ indicating a critical balance between nutrient supply and cell factors. (D) A cubic presentation of the Box-Behnken experimental design showing experimental cell culture outcomes for run conditions for cell recovery $\left(\mathrm{Y}_{2}\right.$, note transformed data) as a function of the three factors: $X_{S^{\prime}} X_{M V}$ and $X_{F T}$.

$\mathrm{X}_{\mathrm{FT}}$ : Media exchange time; $\mathrm{X}_{\mathrm{MV}}$ : Media volume; $\mathrm{X}_{\mathrm{SD}}$ : Seeding density; $\mathrm{Y}_{2}$ : Harvest population doublings (24 h postexperiment population doubling).

the cells recover relatively well after the experiment, irrespective of the other parameter values. Figure 3C shows two regions of maximum response when the $\mathrm{X}_{\mathrm{FT}}$ is set at a high value (i.e., midprocess); there is substantially greater cell recovery after an experimental run with low media levels combined with low $\mathrm{X}_{\mathrm{SD}}$ or with high media levels and high $\mathrm{X}_{\mathrm{SD}}$ (the former having greater effect). The low media and cell input condition is preferable based on cost-optimization criteria. Figure 3D shows the experimental value achieved for each run condition that was used to generate the process model.

The cell recovery model predicts that highyield conditions $\left(\mathrm{Y}_{1}\right)$ do not simply correlate with low subsequent cell growth, so the effects on postexperiment recovery are not simply mediated by the cell density reached in the experimental period. It also indicates that a very high rate of recovery of cells can be achieved after a singlecell suspension passage method, but that this recovery is very sensitive to the preceding culture conditions. This is of particular interest given the number of chemical mediators (i.e., ROCK inhibitors) currently being investigated to overcome the problem of cell death post-singlecell passage [16]. The variables investigated are clearly key determinants of this outcome and indicate that a full characterization of the process design space for simple bioprocessing parameters may be able to change process outcomes as effectively as further chemical modifications of the culture milieu. The model is consistent with the conclusion that when the cell cultures fall 


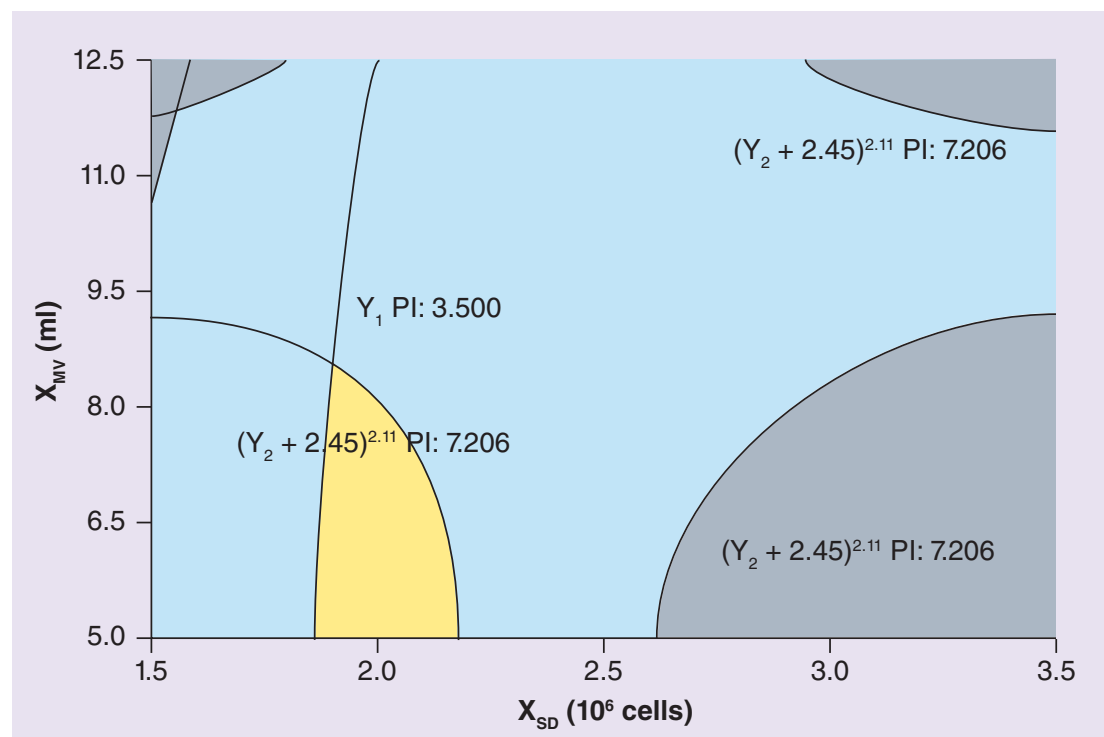

Figure 4. Multiple response overlay graph comprising superimposed contour plots from each response; passage yield and harvest population doubling. Regions that do not fit the optimization criteria are shaded grey. Interval estimates are added to the graphical optimization to account for uncertainty in the point predictions and impact of uncertainty on achieving process goals. The blue region corresponds to where the point estimate meets the criteria requirements, but part of an interval estimate does not. The yellow region shows where the entire range of all intervals meets the specified criteria and defines window(s) of operability, or the 'sweet spot'.

$\mathrm{PI}$ : Prediction interval; $\mathrm{X}_{\mathrm{MV}}$ : Media volume; $\mathrm{X}_{\mathrm{SD}}$ : Seeding density; $\mathrm{Y}_{1}$ : Passage yield; $Y_{2}$ : Harvest population doubling. beneath a certain threshold of media availability per cell, subsequent postpassage recovery will be very slow. This could either be because the cells are already committed to death, they have a prolonged inhibition of proliferation or they are more sensitive to the stress of the passage process. In any case, it indicates that multivariate empirical models such as these are essential to achieve both optimal proliferative performances over subsequent passages and to achieve process robustness. Although primarily critical for robust manufacture, this is also an important point for experimental scientists where a lack of awareness and control of such sensitivity will jeopardize the reproducibility of data.

Table 4. The optimal process performance predicted by the models and the corresponding validation data from a cell culture run.

\begin{tabular}{|c|c|c|}
\hline Response & Predicted response $(95 \% \mathrm{Cl})$ & Actual response \\
\hline$Y_{1}\left(\times 10^{6}\right.$ cells $)$ & $5.27(3.47-7.08)$ & $5.01 \pm 0.35$ \\
\hline$Y_{2}$ & $0.43(0.12-0.71)$ & $0.43 \pm 0.13$ \\
\hline \multicolumn{3}{|c|}{$\begin{array}{l}\text { The model generated from the experimental data predicted new operating conditions to achieve } \\
\text { optimal response values for } Y_{1} \text { and postexperimental conditions for cell recovery }\left(Y_{2} ; \text { i.e., for }\right. \\
\text { maximizing these selected responses and reducing process cost). These conditions were: seeding } \\
\text { density set at } 2.0 \times 10^{6} / T 25, \text { media volume set at } 6.0 \mathrm{ml} / \mathrm{T} 25 \text { and media exchange set at } 24 \mathrm{~h}(-12) \text {. } \\
\text { The actual response shows that a validation cell culture run conducted in the laboratory fell within } \\
\text { the predicted range for both of the modeled responses, confirming the utility of the models for } \\
\text { optimizing process responses. } \\
Y_{1} \text { : Cell yield; } Y_{2} \text { : Harvest population doublings. }\end{array}$} \\
\hline
\end{tabular}

Optimum conditions for the unit of manufacture were calculated using the mathematical multiple-response optimization routine (desirability analysis) in Design Expert. The design specification aimed to achieve maximum yield $\left(Y_{1}\right)$ from the experimental run conditions and maximum recovery $\left(\mathrm{Y}_{2}\right)$ in the first $24 \mathrm{~h}$ of subsequent culture. Superimposing critical response contours generated a graphical optimization display showing the two regions where these critical properties are simultaneously met (Figure 4 ). The model optimization criteria were set to minimize $\mathrm{X}_{\mathrm{MV}}$, media exchange frequency and input cell numbers. A lower weighting was given to feed frequency due to the capacity of an automated system to conduct this step without manual labor. The optimization criteria resulted in predicted optimal factor settings of $2 \times 10^{6} / \mathrm{T} 25$ cell seeding, $6 \mathrm{ml} / \mathrm{T} 25$ culture media and a media exchange after $24 \mathrm{~h}$. Using the point prediction node in Design Expert, the expected responses and associated interval estimates were calculated based on the prediction equation shown in the analysis of variance output. Table 4 shows the process outputs predicted by the models for these process parameters and the actual process outputs achieved from a verification cell culture run. The average response from the validation experiment, comprising three runs at the specified combination of factors settings, was within the bounds of the prediction interval, indicating that the prediction represents the true response surface.

\section{Discussion}

Creating manufacturing processes that can reduce the cost of goods and demonstrate robustness to product regulators will be necessary for the commercial viability and product approval of many cell therapies. The described approach is an example of how confidence can be built, subprocess by subprocess, into a full manufacturing process. This is compatible with a 'quality-by-design' approach to achieve scientific understanding of process control, a system that is being increasingly encouraged by medical product regulators to improve the reproducibility of product safety and efficacy. As such studies are applied in the real manufacturing environment, longer-term validation will be required to show stability over time and throughout longer processes. More knowledge will need to be developed regarding the intermediate process parameters (and associated monitoring measurements) that form the inputs and outputs of the subprocesses 
or units of manufacture that together generate a complete system. In this instance, important quality factors, such as genetic stability and pluripotency markers, were not measured as outputs of the model, as meaningful changes in such quality would not be expected to develop over a single experimental passage period. For the purposes of selecting operating conditions from these models, we have made an assumption that a cell population that recovers faster after passage has been less stressed and is more likely to retain desirable characteristics in the long term. However, as a deeper understanding of the parameters that aid the retention of cell phenotype or contribute to clinical efficacy is developed, this will enable further process responses or associated criteria to be incorporated into the models.

The conditions in Table 4, validated with experimental cell cultures, represent a $31 \%$ reduction in cell culture media use, a $25 \%$ reduction in process time and a $20 \%$ reduction in input cells over the experimental passage period relative to the central or standard operating conditions. Although the validated conditions also deliver a $44 \%$ reduction in absolute $\mathrm{Y}_{1}$ per passage relative to the standard process, this is compensated by the 0.4 population doublings achieved over the first $24 \mathrm{~h}$ of the subsequent growth period (relative to zero growth under central conditions). If we had not modeled this 'cell recovery' response, we would have selected criteria for passage conditions that resulted in significant cell death in subsequent processing. This is an important note of caution for giving appropriate consideration to key process responses before embarking on optimization. Although passaging cells at lower density would also have a cost overhead in terms of space and flask requirements, this added complexity was not considered for the purposes of this exercise. Such considerations could be accounted for by incorporating additional measured outputs in the experimental designs and generating additional models that must be satisfied by the selected process operating parameters. In this manner, the methods can be customized for a specific production case.

This work was conducted on a single cell line as this is representative of the manufacturing scenario, which will usually be based on a defined single cell line bank. Absolute values predicted by the models reported here will be highly sensitive to the strict operating controls placed on the process (i.e., they are only valid if the control environment is specified); a product developer can apply a similar methodology to specify operating conditions for their banked cell line, within their controlled environment, to their own quality specification. This is parallel to manufacturing in other industries; we would not anticipate being able to take defined material from one manufacturer (i.e., other cell lines/banks) and location through another manufacturer's processes and achieve the same output. Therefore, the value here is not the precise operating values reported, but the evidence that a relatively simple combination of control and experimental design can accurately predict a chosen aspect of culture performance. It is reasonable to anticipate that industrial processes will achieve similar benefits from using these approaches to model relevant process variables for their case-specific critical process responses.

\section{Conclusion}

This application of RSM design of experiments shows that empirical models can be generated and used predictively to optimize outputs in a particularly challenging and industrially relevant human cell culture system, and that they will be an increasingly important tool in creating robust and economical manufacturing processes for cellular therapies. Systematic design of experiment approaches will need to be widely applied to design fit-for-purpose manufacturing processes and systems for cellular therapies.

\section{Executive summary}

- The effective application of a quality-by-design process development tool - response surface method - to a subprocess of embryonic stem cell manufacture is demonstrated.

- Significant reduction in embryonic stem cell manufacturing costs are achieved using a scalable methodology.

- Operating conditions that achieve significant passage yield and rapid cell recovery postpassage without the use of a ROCK inhibitor are identified.

Predicted optimal operating conditions are validated in confirmatory runs.

- Insight is provided into cell sensitivity to manufacturing conditions, the interaction of manufacturing parameters and the consequent control required to achieve critical process outputs.

- The necessity of combining multiple models using carefully selected quality outputs for optimization is identified and discussed. 


\section{Financial \& competing interests disclosure}

This work was supported by the UK Engineering and Physical Sciences Research Council as part of the remedi Grand Challenge and the EPSRC Centre for Innovative Manufacturing in Regenerative Medicine, which is a partnership of Loughborough, Nottingham and Keele Universities, and industry and public sector stakeholders. The authors have no other relevant affiliations or financial involvement with any organization or entity with a financial interest in or financial conflict with the subject matter or materials discussed in the manuscript apart from those disclosed.

No writing assistance was utilized in the production of this manuscript.

\section{Ethical conduct of research}

The authors state that they have obtained appropriate institutional review board approval or have followed the principles outlined in the Declaration of Helsinki for all human or animal experimental investigations. In addition, for investigations involving human subjects, informed consent has been obtained from the participants involved.

\section{References}

Papers of special note have been highlighted as: - of interest

1 Mimeault M, Hauke R, Batra SK. Stem cells: a revolution in therapeutics - recent advances in stem cell biology and their therapeutic applications in regenerative medicine and cancer therapies. Clin. Pharm. Ther. 82, 252-264 (2007).

2 Mason C, Dunhill P. A brief definition of regenerative medicine. Regen. Med. 3, 1-5 (2008).
3 Williams DJ, Thomas RJ, Hourd PC et al. Precision manufacturing for clinical quality regenerative medicines. Philos. Transact. A Math. Phys. Eng. Sci. 370, 3924-3949 (2012).

- Provides a comprehensive outline of the scalability requirements and manufacturing issues associated with producing the cell stocks for regenerative cell-based medicines.

4 Ratcliffe E, Thomas RJ, Williams DJ. Current understanding and challenges in bioprocessing of stem cell-based therapies for regenerative medicine. Br. Med. Bull. 100, 137-155 (2011).

5 Mason C, Hoare M. Regenerative medicine bioprocessing: building a conceptual framework based on early studies. Tissue Eng. 13, 301-311 (2007).

6 Poon GK, Chan JS, Williams DJ. Experimental study of workpiece-level variability in blind-via electroplating. Proc. Inst. Mech. Eng. 215, 521-530 (2001).

7 Abu-Absi SF, Yang L, Thompson P et al. Defining process design space for monoclonal antibody cell culture. Biotech. Bioeng. 106, 894-905 (2010).

- Provides an outline of the progress in applying advanced statistical experimental designs to the production of biologics, methods that will need to be applied for optimal and consistent cell therapy production.

8 Thomas RJ, Hourd P, Williams DJ. Application of process quality engineering techniques to improve the understanding of the in vitro processing of stem cells for therapeutic use. J. Biotechnol. 136, 148-155 (2008).

9 Kirouac D, Zandstra P. The systematic production of cells for cell therapies. Cell Stem Cell 3, 369-381 (2008).
10 Thomson JA, Itskovitz-Eldor J, Shapiro SS et al. Embryonic stem cell lines derived from human blastocysts. Science 282, 1145-1147 (1998).

11 Mummery CL. Cardiology: solace for the broken-hearted? Nature 433, 585-587 (2005).

12 Denning C, Allegrucci C, Priddle $\mathrm{H}$ et al. Common culture conditions for maintenance and cardiomyocyte differentiation of the human embryonic stem cell lines, BG01 and HUES-7. Int. J. Dev. Biol. 50, 27-37 (2006).

- Describes the search for common methodologies for key therapeutic cell line production.

13 Thomas RJ, Anderson D, Chandra A et al. Automated, scalable culture of human embryonic stem cells in feeder-free conditions. Biotech. Bioeng. 102, 1636-1644 (2009).

- Describes the necessary adaptation of human embryonic stem cell manufacturing processes to scalable and robotic technologies.

14 Hasegawa K, Fujioka T, Nakamura Y, Nakatsuji N, Suemori H. A method for the selection of human embryonic stem cell sublines with high re-plating efficiency after single-cell dissociation. Stem Cells 24, 2649-2660 (2006).

- Describes the quality issues that can arise when cell cultures are adapted to different formats.

15 Anderson MJ, Whitcomb PJ. RSM Simplified: Optimizing Processes Using Response Surface Methods for Design of Experiments. Productivity Press, NY, USA (2005).

16 Watanabe K, Ueno M, Kamiya D et al. A ROCK inhibitor permits survival of dissociated human embryonic stem cells. Nat. Biotech. 25, 681-686 (2007). 